\title{
Intervenção Coronária Percutânea Ad Hoc na Angina Estável: Reflexões Sobre Uma Prática Segura
}

Ver artigo relacionado
na página 196

\author{
Lino Patrício ${ }^{1}$
}

N artigo "Segurança e Eficácia da Intervenção Coronária Percutânea Ad Hoc em Pacientes com Angina Estável", de autoria de Carneiro et al. ${ }^{1}$, é apresentado o mais recente estudo randomizado comparando intervenção coronária percutânea ad hoc e intervenção coronária percutânea estagiada na doença coronária estável, tendo como desfechos morte, infarto agudo do miocárdio, acidente vascular cerebral, cirurgia e complicações hemorrágicas. A intervenção coronária percutânea ad hoc teve início nos casos de síndrome coronária aguda com supradesnivelamento do segmento ST, sendo, posteriormente, utilizada também nos casos sem supradesnivelamento do segmento ST, em que a lesão culpada pela isquemia passou a ser tratada concomitantemente à cinecoronariografia. O procedimento foi transposto para a doença estável, embora esse grupo de pacientes apresente alguns aspectos particulares que precisam ser avaliados.

O primeiro aspecto refere-se à segurança relacionada às complicações da intervenção coronária percutânea, assim como, conforme mencionado pelos autores, ao risco de nefropatia e de radiação. Como ficou demonstrado, nesse artigo e em estudos anteriores, a intervenção coronária percutânea ad hoc é segura quanto aos desfechos morte, acidente vascular cerebral, cirurgia e infarto agudo do miocárdio, existindo benefício no que se refere às complicações hemorrágicas. Sabemos hoje que essas complicações, quando consideradas maiores, também têm influência no prognóstico. Esse estudo evidenciou claramente que os níveis de creatinina sérica não demonstraram diferença significativa entre os dois grupos estudados. Devese, no entanto, ressaltar que $97 \%$ desses pacientes apresentavam função renal com níveis de creatinina inferiores a 1,5 mg/dl, precisando ainda ser esclarecido se a coronariografia seguida de intervenção coronária percutânea é igualmente segura nos doentes com função renal comprometida. Os tempos de radiação dos procedimentos administrados em poucos dias, por sua vez, devem ser considerados em conjunto, pelo fato de os efeitos da radiação serem cumulativos. No entanto, atualmente, os tempos de radiação estão reduzidos, à exceção dos procedimentos prolongados, como as oclusões totais, devendo-se considerar a adoção de outra estratégia para esse subgrupo de pacientes.

$\mathrm{Na}$ doença coronária estável, e principalmente quando existe doença multiarterial (cerca de 30\% nos dois grupos estudados), nem sempre é possível definir a lesão culpada pela isquemia e, portanto, pelos sintomas anginosos. Muitos desses pacientes apresentam história de infarto (13\% nessa série), o que torna necessário definir as áreas de viabilidade.

Recentemente, com a publicação do estudo SYNergy between percutaneous coronary intervention with TAXus and cardiac surgery $(\text { SYNTAX })^{2}$, definiram-se escores angiográficos que podem facilitar, de forma ad hoc, a decisão pela intervenção coronária percutânea imediatamente após a coronariografia. A intervenção coronária percutânea ad hoc não pode, só por esse fato e nesse grupo de pacientes com doença estável, ultrapassar os critérios de revascularizar apenas lesões isquêmicas e intervir em qualquer lesão angiográfica, independentemente de ser ou não provocadora de isquemia. Sabia-se já, e o estudo Clinical Outcomes Utilizing Revascularization and Aggressive Drug Evaluation (COURAGE) ${ }^{3}$ confirmou, que o tratamento médico otimizado pode ser tão bom como a intervenção coronária percutânea nos pacientes com lesões sem isquemia significativa ${ }^{4}$. Nos pacientes multiarteriais, em que se toma a decisão ad hoc de em quais lesões intervir, o estudo Fractional Flow Reserve Versus Angiography for Multivessel Evaluation (FAME) ${ }^{5}$, recentemente publicado, demonstrou que a utilização da ferramenta "reserva de fluxo fracionada" ajuda na decisão e tem significado prognóstico ${ }^{6}$.

Por último, e não menos importante, deve-se levar em conta o aspecto não só da comodidade para

\footnotetext{
1 Laboratório de Hemodinâmica do Hospital de Santa Marta - Lisboa, Portugal.

Correspondência: Lino Patrício. Rua Nóbrega e Sousa, 1/14 a C - Lisboa, Portugal - 1750

E-mail: linopatricio@gmail.com

Recebido em: 3/6/2009 • Aceito em: 4/6/2009
} 
o paciente, mas também de seu envolvimento na decisão sobre a intervenção coronária percutânea. O fato de a intervenção coronária percutânea ser ad hoc não implica que o paciente não deva ter conhecimento da decisão ou que não possa participar dela. Mesmo ad hoc, a intervenção coronária percutânea carece de alguns minutos de reflexão tanto por parte do paciente como por parte do operador.

Após o excelente trabalho aqui analisado e a confirmação resultante da prática clínica atual, conclui-se que a intervenção coronária percutânea ad hoc é um procedimento seguro e cômodo, desde que salvaguardados os aspectos mencionados.

\section{CONFLITO DE INTERESSES}

O autor declarou inexistência de conflito de interesses.

\section{REFERÊNCIAS BIBLIOGRÁFICAS}

1. Carneiro JKR, Carneiro JD Neto, Lima Neto JA, Parente FL, Aguiar LS, Rocha BAM, et al. Segurança e eficácia da intervenção coronária percutânea ad hoc em pacientes com angina estável. Rev Bras Cardiol Invas. 2009;17(2):196-201.

2. Serruys PW, Morice MC, Kappetein AP, Colombo A, Holmes DR, Mack MJ, et al. Percutaneous coronary intervention versus coronary-artery bypass grafting for severe coronary artery disease. N Engl J Med. 2009;360(10):961-72.

3. Boden WE, O'Rourke RA, Teo KK, Hartigan PM, Maron DJ, Kostuk WJ, et al. Optimal medical therapy with or without PCI for stable coronary disease. N Engl J Med. 2007;356(15): 1503-16.

4. Shaw LJ. Differential improvement in stress myocardial perfusion ischemia following percutaneous coronary intervention as compared with optimal medical therapy alone: Nuclear substudy results from the Clinical Outcomes Utilizing Revascularization and Aggressive Drug Evaluation (COURAGE) trial. Late-breaking clinical trials. Orlando: American Heart Association 2007 Scientific Sessions; 2007.

5. Tonino PA, De Bruyne B, Pijls NH, Siebert U, Ikeno F, van' t Veer $M$, et al. Fractional flow reserve versus angiography for guiding percutaneous coronary intervention. $N$ Engl J Med. 2009;360(3):213-24.

6. Ellis SG. Refining the art and science of coronary stenting. N Engl J Med. 2009;360(3):292-4. 